\title{
Diffuse optical measurements of head and neck tumor hemodynamics for early prediction of chemoradiation therapy outcomes
}

Lixin Dong

Mahesh Kudrimoti

Daniel Irwin

Li Chen

Sameera Kumar

Yu Shang

Chong Huang

Ellis L. Johnson

Scott D. Stevens

Brent J. Shelton

Guoqiang Yu 


\title{
Diffuse optical measurements of head and neck tumor hemodynamics for early prediction of chemoradiation therapy outcomes
}

\author{
Lixin Dong,, ${ }^{a}$ Mahesh Kudrimoti, ${ }^{b}$ Daniel Irwin, ${ }^{a}$ Li Chen, ${ }^{c, d}$ Sameera Kumar, ${ }^{b}$ Yu Shang, ${ }^{\text {a }}$ Chong Huang, ${ }^{a}$ \\ Ellis L. Johnson, ${ }^{b}$ Scott D. Stevens, ${ }^{e}$ Brent J. Shelton, ${ }^{c, d}$ and Guoqiang Yu ${ }^{a, *}$ \\ aUniversity of Kentucky College of Engineering, Department of Biomedical Engineering, 143 Graham Avenue, Lexington, Kentucky 40506, United \\ States \\ bUniversity of Kentucky College of Medicine, Department of Radiation Medicine, 800 Rose Street, Lexington, Kentucky 40536, United States \\ 'University of Kentucky, Biostatistics and Bioinformatics Shared Resource Facility, Markey Cancer Center, Lexington, 800 Rose Street, Kentucky \\ 40536, United States \\ dUniversity of Kentucky College of Public Health, Department of Biostatistics, Lexington, 111 Washington Avenue, Kentucky 40536, United States \\ eUniversity of Kentucky College of Medicine, Department of Radiology, 800 Rose Street, Lexington, Kentucky 40536, United States
}

\begin{abstract}
This study used a hybrid near-infrared diffuse optical instrument to monitor tumor hemodynamic responses to chemoradiation therapy for early prediction of treatment outcomes in patients with head and neck cancer. Forty-seven patients were measured once per week to evaluate the hemodynamic status of clinically involved cervical lymph nodes as surrogates for the primary tumor response. Patients were classified into two groups: complete response $(\mathrm{CR})(n=29)$ and incomplete response $(\mathrm{IR})(n=18)$. Tumor hemodynamic responses were found to be associated with clinical outcomes (CR/IR), wherein the associations differed depending on human papillomavirus (HPV-16) status. In HPV-16 positive patients, significantly lower levels in tumor oxygenated hemoglobin concentration $\left(\left[\mathrm{HbO}_{2}\right]\right)$ at weeks 1 to 3 , total hemoglobin concentration at week 3 , and blood oxygen saturation $\left(\mathrm{StO}_{2}\right)$ at week 3 were found in the IR group. In HPV-16 negative patients, significantly higher levels in tumor blood flow index and reduced scattering coefficient $\left(\mu_{\mathrm{s}}^{\prime}\right)$ at week 3 were observed in the IR group. These hemodynamic parameters exhibited significantly high accuracy for early prediction of clinical outcomes, within the first three weeks of therapy, with the areas under the receiver operating characteristic curves (AUCs) ranging from 0.83 to 0.96 . $\odot 2016$ Society of Photo-Optical Instrumentation Engineers (SPIE) [DOI: 10 .1117/1.JBO.21.8.085004]
\end{abstract}

Keywords: near-infrared; diffuse optics; head and neck cancer; papilloma virus status; chemoradiation therapy; tumor hemodynamics. Paper 160398R received Jun. 11, 2016; accepted for publication Aug. 8, 2016; published online Aug. 26, 2016.

\section{Introduction}

Head and neck cancer accounts for about $3 \%$ to $5 \%$ of all cancers in the United States. ${ }^{1}$ More than $90 \%$ of head and neck cancers arise from the epithelium lining the sinonasal tract, oral cavity, pharynx (oropharynx, nasopharynx, hypopharynx), and larynx and show microscopic evidence of squamous differentiation. Head and neck squamous cell carcinoma (HNSCC) frequently spreads to the lymph nodes in the neck. The major risk factors for HNSCCs are tobacco use, alcohol consumption, and infection with human papillomavirus (HPV). ${ }^{2} \mathrm{HPV}$ has been found in head and neck cancers from all sites with a higher prevalence in oropharynx cancer. ${ }^{3}$ In clinical practice, HPV-16 immunohistochemistry is recommended for the detection of HPV DNA in tumor cell nuclei.

Radiation therapy is a principal modality in the treatment of HNSCC and may be combined with chemotherapy, surgery, or both according to the stage, size, and location of tumors. Radiation therapy works by damaging the DNA of cancer cells, ultimately resulting in cell death. The treatment can also cause long-term changes in the vasculature mediated through inflammatory cytokines and other agents. ${ }^{4}$ When using X-ray beams for therapy, the radiation effect is manifest mainly through an indirect mechanism involving free radical formation within the intracellular matrix that attacks tumor cells. ${ }^{5}$ The efficacy of X-ray radiation therapy is known to be dependent on tumor oxygen status. Hypoxic tumor cells can repair their DNA damage more readily than euoxic cells where the damage is irreversible because of the interaction of tissue oxygen with free radicals. ${ }^{6,7}$ Many tumors are hypoxic because of abnormal vasculature, malignancy-related anemia, and/or high oxygen consumption by tumor cells. ${ }^{7}$ Studies using polarographic electrodes have shown pretreatment tumor hypoxia are associated with significantly poor responses to radiation therapy when compared to oxygenated tumors. ${ }^{8-11}$ However, in these studies, some well-oxygenated tumors failed to respond while some hypoxic tumors responded well, possibly due to the dynamic changes during treatment in tumor oxygen status induced by radiation. Therefore, repeated monitoring of individual tumor hemodynamic status during therapy may provide predictive information for treatment outcomes.

Tumor hemodynamics, however, are not routinely monitored over the course of cancer therapy in clinical practice due to the lack of appropriate technologies. Several methods exist for the measurement of tissue oxygenation or blood flow in tumors. The invasive polarographic electrode method provides a point measurement of tumor oxygenation, ${ }^{8-11}$ but tends to produce 
inconsistent results in highly heterogeneous tissues. Imaging modalities, such as positron emission tomography (PET), ${ }^{12}$ dynamic computed tomography (CT), ${ }^{13}$ and dynamic magnetic resonance imaging (MRI), ${ }^{14}$ provide structural and functional information about tumors, but require large and costly instrumentation that limits their potential for routine use in clinic. Moreover, some of these techniques (e.g., CT and PET) expose patients to ionizing radiation.

Near-infrared (NIR) diffuse optical methods offer a noninvasive, portable, fast, and low-cost alternative for frequent monitoring of tumor microvascular hemodynamics at the bedside over the time course of treatment. ${ }^{15-18}$ For example, Sunar et al. previously used a hybrid NIR diffuse optical instrument for noninvasive measurements of tumor hemodynamic responses to chemoradiation therapy in a small group of patients with HNSCC. ${ }^{15}$ Tumors exhibited significant dynamic flow and oxygenation changes during the first four weeks of the treatment. However, their study was limited by the small number of patients examined $(n=8)$ and only one patient out of 8 showed a partial response to the treatment. It was, therefore, impossible to draw a conclusion from this pilot study about the feasibility of measuring tumor hemodynamics to predict treatment outcomes.

Our study aimed to investigate the accuracy of weekly hemodynamic measurements in HNSCCs during chemoradiation therapy for the prediction of treatment outcome in a relatively large population $(n=47)$. Previously, we have developed and validated a hybrid diffuse optical instrument combining a commercial frequency-domain NIR tissue oximeter (Imagent, ISS Inc., IL) with a custom-designed diffuse correlation spectroscopy (DCS) flowmeter, which allowed for simultaneous quantification of blood flow and oxygenation in deep tissues up to several centimeters. ${ }^{17-20}$ While access to the primary tumor volume of HNSCC is not always possible with this device due to the limit of tumor location (e.g., inside oral cavity), we measured instead clinically involved, shallow cervical lymph nodes as surrogates for primary tumor responses because both primary tumors and cervical lymph nodes were included in the radiation treatment volume and a previous study showed that primary tumors and their regional lymph node metastases in HNSCC had comparable patterns of oxygenation. ${ }^{21}$ We monitored hemodynamic characteristics of these cervical lymph nodes once per week over the chemoradiation treatment period of 7 weeks and investigated the accuracy of using tumor hemodynamic responses to therapy for predicting one-year treatment outcomes based on the receiver operating characteristic (ROC) curve and the area under the ROC curve (AUC).

\section{Methods and Materials}

\subsection{Hybrid Diffuse Optical Instrument for Monitoring Tumor Hemodynamics}

Our hybrid diffuse optical instrument combining a commercial NIR tissue-oximeter (Imagent, ISS, IL) and a custom-designed DCS flowmeter allows us to simultaneously measure tumor blood flow and blood oxygenation (Fig. 1). The commercial Imagent system used for tumor blood oxygenation measurement is a spatially resolved frequency-domain system employing 16 laser diodes at 690, 750, 780, and $830 \mathrm{~nm}$ (four diodes for each wavelength) modulated at $110 \mathrm{MHz}$ as sources and one photomultiplier tube (PMT) as the detector. ${ }^{22}$ Tumor oxygenation is measured using a fiber-optic probe consisting of 16 source fibers coupled to the 16 laser diodes and one detector fiber connected to the PMT [Fig. 1(a)]. The source fibers (diameter $=400 \mu \mathrm{m})$ are arranged at distances of $2.0,2.5,3.0$, and $3.5 \mathrm{~cm}$ from the detector fiber (diameter $=2.5 \mathrm{~mm}$ ). The Imagent system measures the reduced amplitudes and phase shifts of the modulated light at the four wavelengths and four source-detector (S-D) distances after the diffused light propagates through the tissue [Fig. 1(b)]. ${ }^{20,22}$ An analytical homogenous solution based on semi-infinite tissue geometry for photon diffusion equation exposes linear relationships between the phases/logarithmic amplitudes and the S-D distances. By fitting the slopes of these linear relationships using the spatially resolved method, tissue absorption coefficient $\left(\mu_{\mathrm{a}}\right)$ and reduced scattering coefficient $\left(\mu_{\mathrm{s}}^{\prime}\right)$ can be extracted at each of four wavelengths. ${ }^{22}$ The tissue blood oxygenation including oxygenated-, deoxygenated-, and total-hemoglobin concentrations (i.e., $\left[\mathrm{HbO}_{2}\right],[\mathrm{Hb}]$, and $\left.\mathrm{THC}=\left[\mathrm{HbO}_{2}\right]+[\mathrm{Hb}]\right)$ and blood oxygen saturation $\left(\mathrm{StO}_{2}=\right.$ $\left[\mathrm{HbO}_{2}\right] / \mathrm{THC} \times 100 \%$ ) can be then calculated from the measured $\mu_{\mathrm{a}}$ at the four wavelengths. ${ }^{20}$ The commercial Imagent system has been previously used for the quantification of tumor optical properties and oxygenation status. ${ }^{17,23}$

Details about DCS theory and instrumentation for tissue blood flow measurements have been described elsewhere. ${ }^{17,24-26}$ Briefly, a laser diode delivers continuous-wave (CW) light at $785 \mathrm{~nm}$ with long coherence length $(>5 \mathrm{~m})$ through a source fiber (diameter $=200 \mu \mathrm{m})$ into the tissue. The motions of moving scatterers (primarily red blood cells in tissue microvasculature) cause light intensity fluctuations that are collected by a single-mode detector fiber (diameter $=5 \mu \mathrm{m}$ ) placed on the tissue surface within a certain distance from the source. The S-D distance for DCS measurement used in this study is $2.5 \mathrm{~cm}$ [Fig. 1(b)]. A single-photon-counting avalanche photodiode (APD) connected to the detector fiber detects the temporal light intensity fluctuation in a single speckle area on the tissue surface and the APD output is sent to an autocorrelator board for calculating the normalized light intensity temporal autocorrelation function, $g_{2}(\tau)=I(t) \cdot I(t+\tau) / I(t)^{2}$, where $\tau$ is the autocorrelation delay time. The normalized electric field temporal autocorrelation function $\left(g_{1}\right)$ can be derived from the measured $g_{2}$ using the Siegert equation ${ }^{27}$

$g_{2}(\tau)=1+\beta\left|g_{1}(\tau)\right|^{2}$

where $\beta$ is a constant determined primarily by the collection optics of the experiment. The unnormalized electric field temporal autocorrelation function $\left(G_{1}\right)$ satisfies the correlation diffusion equation in highly scattering media. ${ }^{24}$ In a semi-infinite homogeneous medium, the analytical solution of correlation diffusion equation can be expressed as ${ }^{24}$

$G_{1}(\rho, \tau)=\frac{v S_{0}}{4 \pi D}\left[\frac{e^{-K(\tau) r_{1}}}{r_{1}}-\frac{e^{-K(\tau) r_{2}}}{r_{2}}\right]$,

where $\rho$ is the source-detector separation, $r_{1}=\sqrt{\rho^{2}+z_{0}^{2}}$, and $r_{2}=\sqrt{\rho^{2}+\left(z_{0}+2 z_{b}\right)^{2}} . \quad K^{2}(\tau)=3 \mu_{\mathrm{a}} \mu_{s}^{\prime}+\mu_{s}^{\prime 2} K_{0}^{2} \alpha\left\langle\Delta r^{2}(\tau)\right\rangle$. $k_{0}=2 \pi n / \lambda$ is the wavenumber of the $\mathrm{CW}$ light in the medium. $z_{0} \approx 1 / \mu_{\mathrm{s}}^{\prime}$ and $z_{b}=\frac{2}{3 \mu_{\mathrm{s}}} \frac{1+R_{\mathrm{eff}}}{1-R_{\mathrm{eff}}}$, where $R_{\mathrm{eff}}$ is the effective reflection coefficient to account for the index mismatch between tissue and air: $R_{\text {eff }}=-1.44 n^{-2}+0.71 n^{-1}+0.668+0.064 n$, and $n$ is the ratio of refraction indices of tissue and air: $n=n_{\text {tissue }} /$ $n_{\text {air }} \simeq 1.33$. The normalized electric field temporal autocorrelation function $g_{1}$ can be obtained from $G_{1}$ 
(a)

(b)
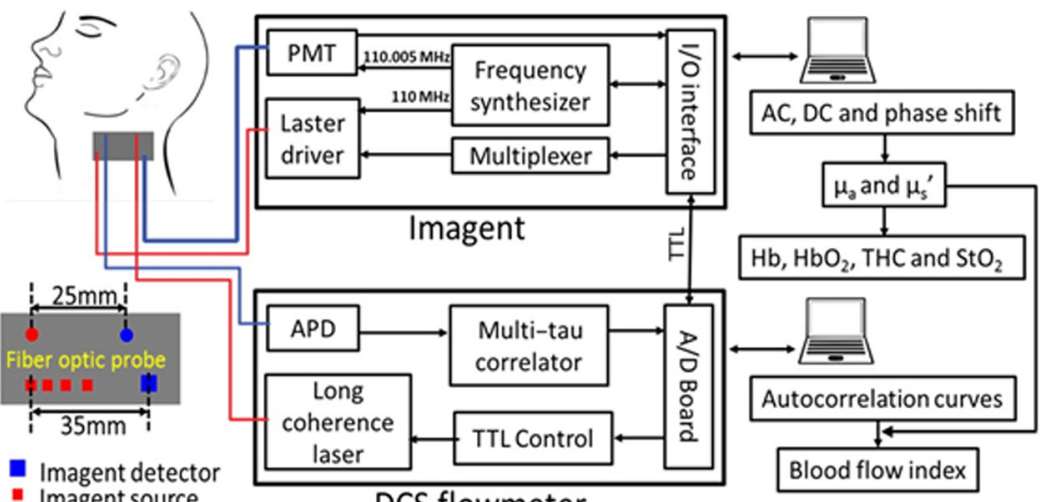

(c)
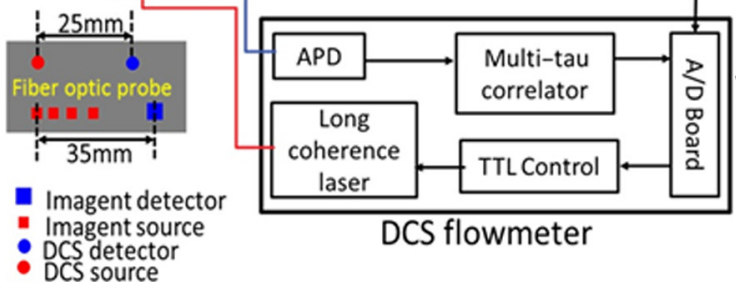

DCS flowmeter

DCS detector

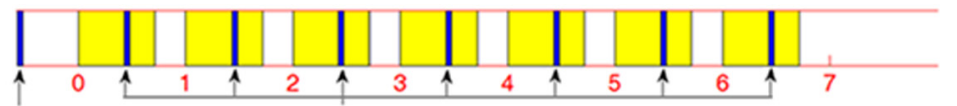
Baseline
Weekly
measurements:
measurements:
Optical measurements Optical measurements
Tumor volume
HPV status

weeks

Therapy follow.

(d)

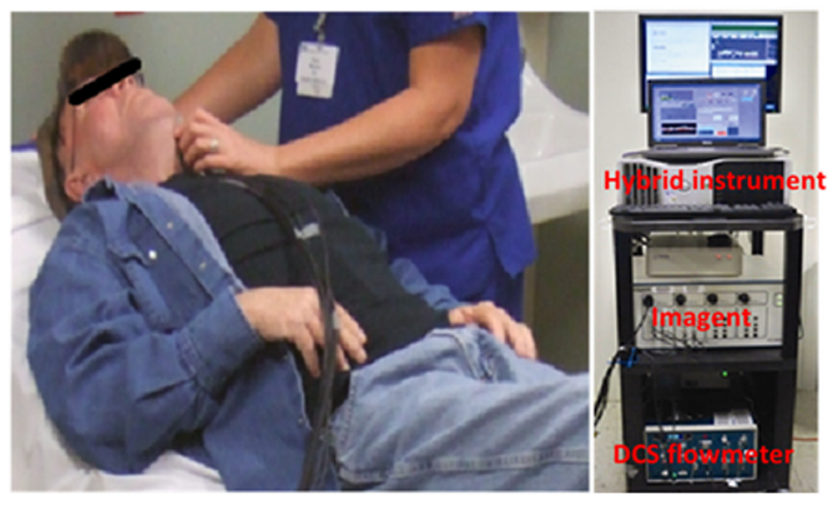

Fig. 1 Hybrid Imagent/DCS instrument for simultaneously measuring tumor oxygenation and blood flow during chemoradiation therapy: (a) schematic of hybrid instrument, (b) fiber-optic probe, (c) chemoradiation therapy protocol and measurement procedure, and (d) optical measurement (left) using a hybrid Imagent/DCS instrument (right).

$$
\begin{aligned}
g_{1}(\rho, \tau) & =\frac{G_{1}(\rho, \tau)}{G_{1}(\rho, 0)} \\
& =\left(\frac{e^{-K(\tau) r_{1}}}{r_{1}}-\frac{e^{-K(\tau) r_{2}}}{r_{2}}\right) /\left(\frac{e^{-K(0) r_{1}}}{r_{1}}-\frac{e^{-K(0) r_{2}}}{r_{2}}\right)
\end{aligned}
$$

For the case of diffusive particle motion, the mean-square displacement $\left\langle\Delta r^{2}(\tau)\right\rangle$ of the moving scatterers in a delay time $\tau$ is $\left\langle\Delta r^{2}(\tau)\right\rangle=6 D_{\mathrm{B}} \tau$, where $D_{\mathrm{B}}$ is an effective diffusion coefficient of the moving scatterers. An $\alpha$ term (0 to 1$)$ is added to account for the fact that not all scatterers in tissue are dynamic and is defined as the ratio of moving scatterers to total scatterers. The combined term $\alpha D_{\mathrm{B}}$ is referred to as the blood flow index (BFI) in biological tissues and is derived by fitting the measured autocorrelation function to the analytical solution of $g_{2}$, based on Eqs. (2) and (3). ${ }^{17,18}$

DCS has been extensively validated against other standards ${ }^{28,29}$ and has been used to detect tumor-to-normal flow contrasts in human breast cancers ${ }^{30,31}$ and to determine hemodynamic effects of radiation therapy on human head and neck cancers. ${ }^{15,16}$

For this study, we constructed a hybrid fiber-optic probe by adding the source and detector fibers for DCS measurement onto the Imagent probe [Fig. 1(b)]. As mentioned earlier, the SD distances for DCS and Imagent were $2.5 \mathrm{~cm}$ and 2.0 to $3.5 \mathrm{~cm}$, respectively, allowing for the detection of a tumor $>2 \mathrm{~cm}$ in size. ${ }^{16}$ Computer-controlled transistor-transistor logic signals were used to control the Imagent and DCS devices working alternately; lasers for DCS and Imagent measurements were turned on sequentially to avoid the light interference between the two measurements [Fig. 1(a)]. The sampling time for a complete frame of blood flow and oxygen measurements is $\sim 4 \mathrm{~s}$ (0. $25 \mathrm{~Hz}$ ).

Our previous investigations have revealed that $\mu_{\mathrm{a}}$ and $\mu_{\mathrm{s}}^{\prime}$ can significantly affect the accuracy of DCS flow quantifications. ${ }^{17,18}$ Thus, $\mu_{\mathrm{a}}$ and $\mu_{\mathrm{s}}^{\prime}$ measured by the Imagent at $780 \mathrm{~nm}$ (close to the 785-nm laser used for DCS flow measurements) were used as inputs for calculating BFI [see Eq. (2)]. The final outputs from this hybrid instrument included $\mu_{\mathrm{a}}, \mu_{\mathrm{s}}^{\prime}$, $\left[\mathrm{HbO}_{2}\right],[\mathrm{Hb}], \mathrm{THC}, \mathrm{StO}_{2}$, and BFI. 


\subsection{Patient Characteristics and Treatment Protocol}

Fifty-six patients $(n=56)$ with head and neck tumors undergoing chemoradiation therapy participated in this study with the signed consents approved by the University of Kentucky Institutional Review Board. Based on the detection limitation in tumor size for optical measurements, ${ }^{16}$ we included Stage III-IVb SCCHN with a cervical lymph node $>2 \mathrm{~cm}$ in the longitudinal dimension. Tumor location and size were identified by MRI, CT, and/or ultrasound imaging. Patients with supraclavicular adenopathy were not enrolled as there was a possibility of the primary origin from other sites in the chest such as lung or esophagus.

Each patient received daily fractional radiation over 7 weeks. Both primary tumor and cervical lymph node were included in the radiation treatment volume. A total dose of 70 Gy was delivered in once daily fractions of $2 \mathrm{~Gy}$, and in the form of highenergy X-ray beams (6 MV) produced by a medical linear accelerator (Clinac 21EX, Varian Medical Systems). Radiotherapy in combination with traditional chemotherapy was applied on these patients. The cisplatin was given at $100 \mathrm{mg} / \mathrm{m}^{2}$ on Day 1 and Day 22 of radiation. The role of chemotherapy (cisplatin) in head/neck cancer was to increase the incidence of double strand breaks (radiosensitization) and decrease the incidence of metastatic diseases.

\subsection{Experimental Protocols}

Prior to treatment, HPV-16 status was examined by immunohistochemistry for most patients. ${ }^{32}$ The biggest clinically involved cervical lymph node was identified by MRI or CT imaging as the measured target and then the tumor node volume and location were quantified. The optical measurement protocol consisted of a pretreatment measurement as baseline and weekly measurements (once per week) over the entire treatment period of 7 weeks [Fig. 1(c)]. Before each optical measurement, an ultrasound machine (Mindray M5, China) was used to verify the location and size of the tumor node. Prior to each measurement, the Imagent system was calibrated on a solid phantom with known optical properties $\left(\mu_{\mathrm{a}}\right.$ and $\left.\mu_{\mathrm{s}}^{\prime}\right)$.

The optical measurement was then continuously taken by placing the hybrid fiber-optic probe on the skin of the selected lymph node [Fig. 1(d)] for $3 \mathrm{~min}$ with a sampling rate of $0.25 \mathrm{~Hz}$, followed by taking a measurement on the forearm flexor muscle for $2 \mathrm{~min}$ as a control measurement for comparison. We took the control measurements on patients' forearm muscles because their forearms were outside the radiation beam and thus less influenced by radiation treatment. This measurement protocol allowed us to compare the sensitivities of using local tumor hemodynamics and global tissue hemodynamics for the prediction of treatment outcomes. Data obtained over the measurement period were averaged and mean values were used to represent tumor/muscle optical properties and hemodynamics.

Clinical outcome assessments such as tumor volume change, local control, remote metastasis, and/or local recurrence with repeat CT scans were conducted every 3 to 4 months after completion of chemoradiation therapy. Since the majority of treatment failures occur in the first 6 months (up to 80\%), we reported control rates at 12 months even though clinical evaluation of response for further intervention was carried out at 10 to 12 weeks post PET scan. Based on clinical outcomes within 1 year after completing chemoradiation therapy, patients were classified into two groups: (1) a complete response (CR) group with disappearance of all target lesions and (2) an incomplete response (IR) group with appearance of distant metastasis and/or local recurrence.

\subsection{Data Analysis and Presentation}

Although 56 patients were measured, two patients do not have one-year follow-up results of treatment outcome yet. Among these 54 patients with treatment outcomes, two were withdrawn from the study at the request of patients and five did not have pretreatment measurements due to instrument issues. Therefore, 47 patients in total were included for our data analysis (see Table 1).

Table 1 Patient/tumor characteristics based on treatment outcomes (IR versus $\mathrm{CR})^{\mathrm{a}}$

\begin{tabular}{lccc} 
& $\begin{array}{c}\text { All patients } \\
(n=47)\end{array}$ & $\begin{array}{c}\text { CR patients } \\
(n=29)\end{array}$ & $\begin{array}{c}\text { IR patients } \\
(n=18)\end{array}$ \\
\hline Age & & & \\
$\begin{array}{l}\text { mean } \pm \text { SE } \\
\text { (years })\end{array}$ & $57.77 \pm 1.16$ & $58.52 \pm 1.40$ & $56.56 \pm 2.00$ \\
$\begin{array}{l}\text { Gender } \\
\text { Male }\end{array}$ & 40 & $27(93.10)$ & $13(72.22)$ \\
$\begin{array}{l}\text { Female } \\
\begin{array}{l}\text { Primary tumor } \\
\text { site }\end{array}\end{array}$ & 7 & $2(6.90)$ & $5(27.78)$ \\
$\begin{array}{l}\text { Base of tongue } \\
\text { Larynx }\end{array}$ & 17 & $11(44.00)$ & $6(37.50)$ \\
Tonsil & 9 & $5(20.00)$ & $4(25.00)$ \\
$\begin{array}{l}\text { Unknown } \\
\text { primary }\end{array}$ & 15 & $9(36.00)$ & $6(37.50)$ \\
Tumor stage & 6 & 4 & 2 \\
Tx + T0-2 & 19 & $13(44.83)$ & $6(33.33)$ \\
T3-4 & 28 & $16(55.17)$ & $12(66.67)$
\end{tabular}

Tumor node size

mean \pm SE $\quad 39.55 \pm 5.82 \quad 37.42 \pm 5.83 \quad 42.97 \pm 11.89$ $\left(\mathrm{cm}^{3}\right)$

Cutaneous

tissue thickness

mean \pm SE $(\mathrm{mm}) \quad 11.22 \pm 0.92 \quad 11.48 \pm 0.86 \quad 10.79 \pm 1.95$

HPV-16 status

1.00

\begin{tabular}{lccc} 
Positive & 26 & $16(69.57)$ & $10(66.67)$ \\
Negative & 12 & $7(30.43)$ & $5(33.33)$ \\
Unknown status & 9 & 6 & 3 \\
\hline
\end{tabular}

avalues in brackets indicate the percentages of patients calculated by excluding those with unknown status. 
The optical measurements over the entire treatment courses of 7 weeks were not always available for each individual due to patient-related issues such as side effects of treatment, fatigue, noncompliance with chemoradiation regimen or due to time constraints of obtaining measurements and modification of treatment schedules as a consequence of temporary breakdown in radiation therapy equipment. Therefore, we marked the number of valid measurements at each time point in figures. Data obtained beyond 4 weeks of the treatment were also not reported because the majority of tumor nodes became too small (due to the chemoradiation therapy) to be visible/measurable by our optical technique.

Two-sample $t$-tests were used to compare the differences between groups in continuous variables including age, tumor node size, and cutaneous tissue thickness above the tumor. Chi-square or Fisher's exact tests were used in categorical variables including gender, tumor site, tumor stage, and HPV-16 status. To compare the time course tumor hemodynamic responses to therapy between the IR and CR groups and between HPV-16 positive and HPV-16 negative groups, we used linear mixed models ${ }^{33}$ with fixed effects of time (as categorical), group (IR/CR or HPV-16 positive/HPV-16 negative), and their interaction and banded covariance structures. Based on the linear mixed model for each hemodynamic parameter, an overall test was first constructed to assess whether there were differences in that parameter between groups at any time point. In the case of a significant overall test, post hoc pairwise comparisons were then conducted at each time point and the Holm's procedure was used to adjust for multiple comparisons. A $p$-value $<0.05$ was considered significant.

The predictive values of different optical measurement parameters can vary over time. The ROC curves were constructed and the areas under the ROC curves (AUCs) were calculated to evaluate the accuracy of various measured parameters at different weeks for predicting treatment outcomes (CR/IR).
The ROC curve is commonly used to evaluate the accuracy of a continuous marker for the prediction of a binary outcome, and it is constructed by plotting the sensitivity against 1-specifity for all possible thresholds of the marker. The AUC corresponding to an ROC curve is used as an overall measure to quantify the accuracy. The bootstrap method ${ }^{34}$ with 500 bootstrap samples is used to obtain the $95 \%$ confidence interval (CI) of the AUC. A 95\% CI of AUC not including 0.5 indicates a significant result.

The statistical analyses were performed by SAS 9.3 and R software version 3.0.1. Particularly, the ROCR package was used to calculate the AUC.

\section{Results}

The differences in tumor hemodynamic responses to chemoradiation therapy between the IR and CR groups were first compared without considering the effects of HPV-16 status (Sec. 3.1). We then refined comparisons by separating patients into an HPV-16 positive group or an HPV-16 negative group, and reported the results in Secs. 3.2 and 3.3, respectively. Based on these results, we summarized predictive values of the measured parameters for one-year treatment outcomes (Sec. 3.4). Finally, we compared the differences in tumor hemodynamic responses and treatment outcomes between the HPV16 positive and HPV-16 negative groups (Sec. 3.5).

\subsection{Comparison of Tumor Hemodynamic Responses Between the IR and CR Groups in All Patients}

Figure 2 shows the dynamic changes of optical properties and tissue hemodynamics over the treatment period of the first 4 weeks, measured in all tumor nodes $(n=47)$ without considering HPV-16 status. Overall, there were significant differences in $\left[\mathrm{HbO}_{2}\right]$ [Fig. 2(b)], THC [Fig. 2(c)], and $\mathrm{StO}_{2}$ [Fig. 2(f)]

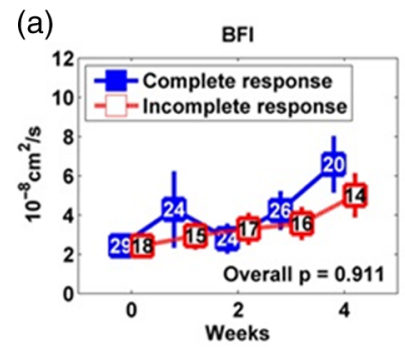

(d) $\mu_{s}^{\prime}$

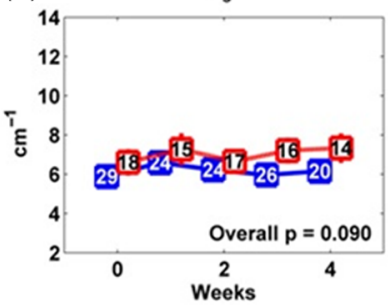

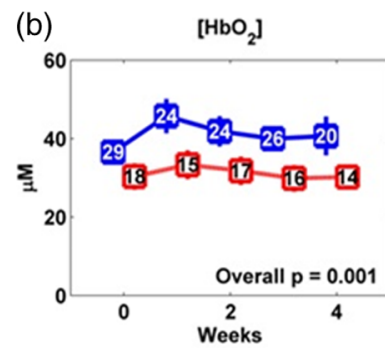

(e)

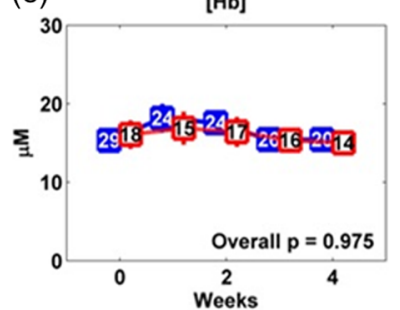

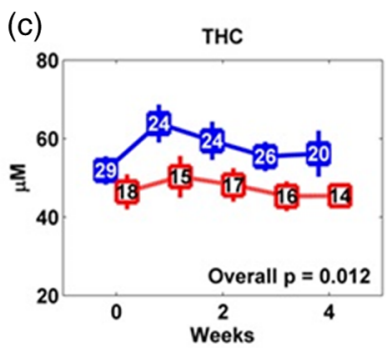

(f)

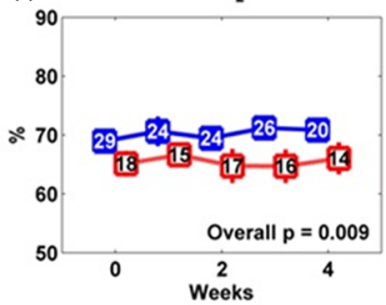

Fig. 2 Averaged values (means \pm standard errors) of hemodynamic and diffuse optical parameters in tumor nodes without considering HPV-16 status grouped by IR and CR: (a) BFI or $\alpha D_{\mathrm{B}}$, (b) oxygenated hemoglobin concentration ( $\left.\left[\mathrm{HbO}_{2}\right]\right)$, (c) THC, (d) reduced scattering coefficient $\left(\mu_{\mathrm{s}}^{\prime}\right)$, (e) deoxygenated hemoglobin concentration $([\mathrm{Hb}])$, and $(\mathrm{f})$ blood oxygen saturation $\left(\mathrm{StO}_{2}\right)$ in tumor nodes at different weeks. Note that $p$-values derived from the linear mixed-effects model for overall differences between the IR and CR groups are shown. The numbers shown at each week represent the valid measurements in IR and CR patients, respectively. 

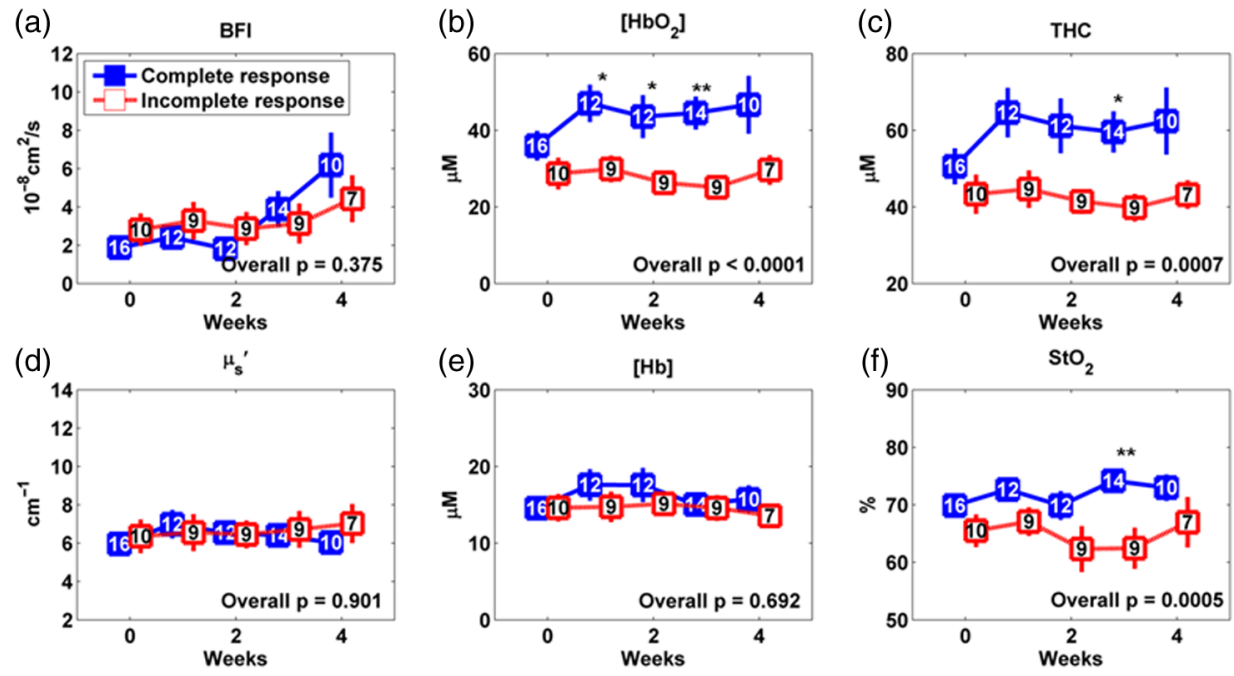

Fig. 3 Averaged values (means \pm standard errors) of hemodynamic and diffuse optical parameters in HPV-16 positive patients grouped by IR and CR: (a) BFI $\left(\alpha D_{\mathrm{B}}\right)$, (b) $\left[\mathrm{HbO}_{2}\right]$, (c) THC, (d) $\mu_{\mathrm{s}}^{\prime}$, (e) [Hb], and (f) $\mathrm{StO}_{2}$ in tumor nodes at different weeks. Note that $p$-values derived from the linear mixed-effects model for overall differences between the IR and CR groups are shown. The numbers shown at each week represent the valid measurements in IR and CR patients, respectively. Significant differences at 0.01 and 0.05 levels between groups derived from pairwise comparisons at different time points are indicated with ${ }^{*}$ and ${ }^{*}$, respectively.

between the IR and CR groups ( $p=0.001,0.012$, and 0.009, respectively). However, post hoc pairwise tests did not find any significant difference in any parameter at any measurement time point between the IR and CR groups.

For control measurements in forearms, no significant differences either over the entire treatment period or at any measurement time point were found between the IR and CR groups (data are not shown).

There were no significant differences between the IR and CR groups prior to the treatment in age, gender, tumor site, tumor stage, tumor node size, cutaneous tissue thickness above the tumor, and HPV-16 status (Table 1).

\subsection{Comparison of Tumor Hemodynamic Responses Between the IR and CR Groups in HPV-16 Positive Patients}

Dynamic changes of optical properties and tissue hemodynamics in tumor nodes of HPV-16 positive patients $(n=26)$ in response to chemoradiation therapy are summarized in Fig. 3. Overall, there were significant differences in $\left[\mathrm{HbO}_{2}\right]$ [Fig. 3(b)], THC [Fig. 3(c)], and $\mathrm{StO}_{2}$ [Fig. 3(f)] between the IR and CR groups ( $p<0.0001, p=0.0007, p=0.0005$, respectively). Post hoc pairwise tests demonstrated that the IR group had significantly lower levels in $\left[\mathrm{HbO}_{2}\right]$ at weeks 1,2 , and 3
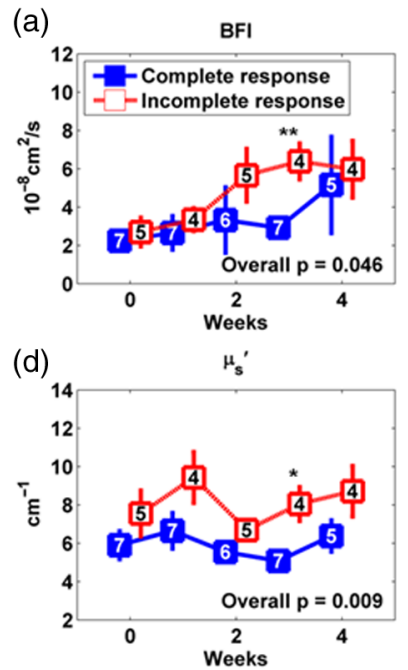

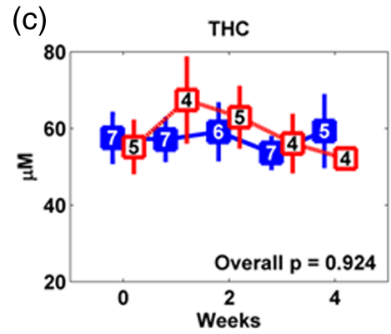

(e)

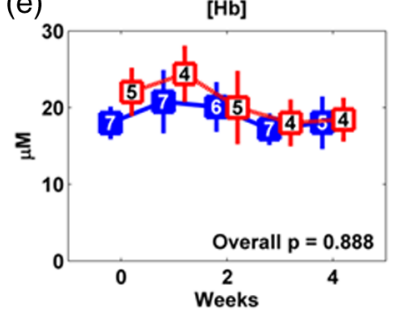

(f)

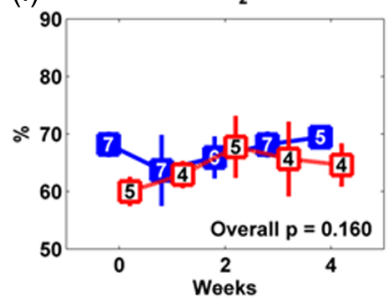

Fig. 4 Averaged values (means \pm standard errors) of hemodynamic and diffuse optical parameters in tumor nodes in HPV-16 negative patients grouped by IR and CR: (a) BFI $\left(\alpha D_{\mathrm{B}}\right)$, (b) oxygenated hemoglobin concentration $\left(\left[\mathrm{HbO}_{2}\right]\right)$, (c) total hemoglobin concentration (THC), (d) reduced scattering coefficient $\left(\mu_{\mathrm{s}}^{\prime}\right),(\mathrm{e})$ deoxygenated hemoglobin concentration $([\mathrm{Hb}])$, and (f) blood oxygen saturation $\left(\mathrm{StO}_{2}\right)$ in tumor nodes at different weeks. Significant differences at 0.01 and 0.05 levels between groups derived from pairwise comparisons at different time points are indicated with ${ }^{*}$ and ${ }^{*}$, respectively. 
Dong et al.: Diffuse optical measurements of head and neck tumor hemodynamics for early prediction...

Table 2 Summary of time-specific AUC estimates (95\% confidence interval) of the measured parameters for the prediction of treatment outcomes (IR or CR) in HPV-16 positive or negative patients. ${ }^{a}$

\begin{tabular}{|c|c|c|c|c|c|}
\hline & Baseline & Week 1 & Week 2 & Week 3 & Week 4 \\
\hline \multicolumn{6}{|c|}{ HPV-16 positive patients } \\
\hline$\left[\mathrm{HbO}_{2}\right]$ & $0.64(0.40,0.83)$ & $0.87(0.68,1.00)$ & $0.83(0.59,0.98)$ & $0.87(0.68,1.00)$ & $0.76(0.49,0.97)$ \\
\hline $\mathrm{THC}$ & $0.62(0.38,0.82)$ & $0.82(0.60,1.00)$ & $0.82(0.60,0.99)$ & $0.83(0.61,0.97)$ & $0.74(0.47,1.00)$ \\
\hline $\mathrm{StO}_{2}$ & $0.66(0.42,0.89)$ & $0.77(0.52,0.96)$ & $0.68(0.42,0.90)$ & $0.84(0.63,0.97)$ & $0.66(0.34,0.94)$ \\
\hline \multicolumn{6}{|c|}{ HPV-16 negative patients } \\
\hline $\mathrm{BFI}$ & $0.57(0.17,0.93)$ & $0.64(0.28,1.00)$ & $0.77(0.43,1.00)$ & $0.93(0.72,1.00)$ & $0.70(0.30,1.00)$ \\
\hline$\mu_{\mathrm{s}}^{\prime}$ & $0.69(0.28,1.00)$ & $0.86(0.57,1.00)$ & $0.80(0.45,1.00)$ & $0.96(0.84,1.00)$ & $0.65(0.18,1.00)$ \\
\hline
\end{tabular}

${ }^{\text {aT }}$ The results italicized are considered to have good predictive ability for treatment outcomes.

( $p=0.02,0.03$, and 0.002 , respectively) [Fig. 3(b)], in THC at week $3(p=0.02)$ [Fig. 3(c)], and in $\mathrm{StO}_{2}$ at week 3 ( $p=0.0004$ ) [Fig. 3(f)], compared to the CR group.

For the control measurements in forearms, no significant differences either over the entire treatment period or at any measurement time point were found between the IR and CR groups (data are not shown).

\subsection{Comparison of Tumor Hemodynamic Responses Between the IR and CR Groups in HPV-16 Negative Patients}

Figure 4 shows the dynamic changes of optical properties and tissue hemodynamics in tumor nodes in response to chemoradiation therapy in HPV-16 negative patients $(n=12)$. Overall, there were significant differences in BFI [Fig. 4(a)] and $\mu_{\mathrm{s}}^{\prime}$ [Fig. 4(d)] between the IR and CR groups ( $p=0.046$ and 0.01 , respectively). Post hoc pairwise tests demonstrated that the mean values of BFI and $\mu_{\mathrm{s}}^{\prime}$ at week $3(p=0.01$ and
0.02 , respectively) in the IR group were significantly higher than those in the CR group.

In HPV-16 negative patients, only forearm $\mathrm{StO}_{2}$ values in the IR group showed an overall significantly lower level than the CR group ( $p=0.04$, data are not shown). However, post hoc pairwise tests did not find any significant difference in forearms at any measurement time point between the IR and CR groups.

\subsection{Accuracy of Tumor Hemodynamic Parameters for Predicting Treatment Outcomes}

To evaluate the accuracy of hemodynamic parameters over the treatment period for the prediction of treatment outcomes, we calculated time-specific ROC curves and AUCs of these optical measurements obtained at different weeks. Table 2 summarizes the ROC results for the parameters showing predictive ability to differentiate IR tumors from CR tumors. Parameters italicized in this table are considered to have good predictive ability for treatment outcomes, with the AUCs ranging from 0.83 to 0.96 . (a) $\left[\mathrm{HbO}_{2}\right]$ at Week 1 for $\mathrm{HPV}$-16 positive

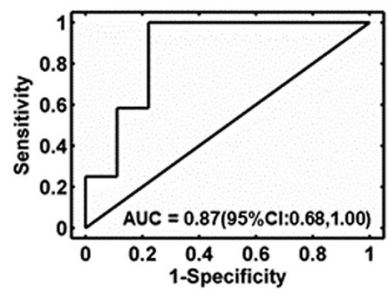

(d) $\mathrm{StO}_{2}$ at Week 3 for $\mathrm{HPV}-16$ positive

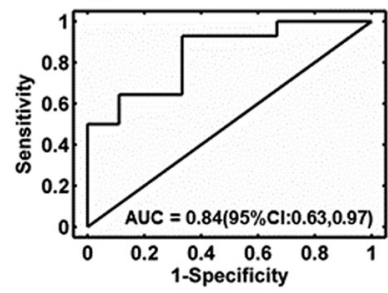

(b) $\left[\mathrm{HbO}_{2}\right]$ at Week 3 for $\mathrm{HPV}-16$ positive

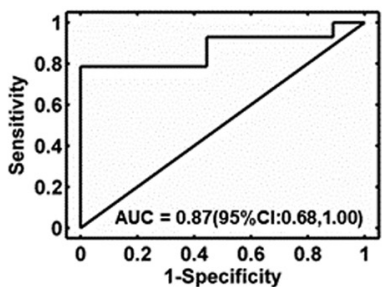

(e) BFI at Week 3 for HPV-16 negative

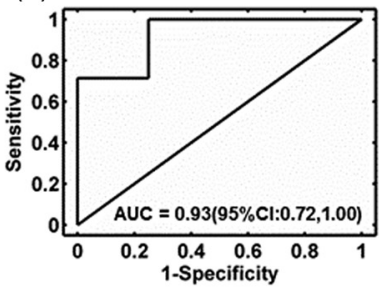

(c) THC at Week 3 for HPV-16 positive

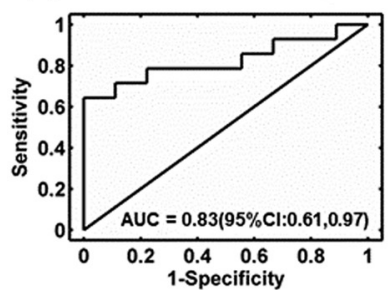

(f) $\mu_{s}^{\prime}$ at Week 3 for HPV-16 negative

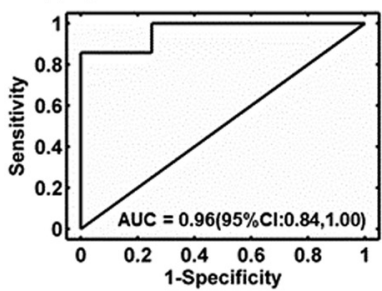

Fig. 5 ROC curves and their associated areas under the curve (AUC) for discriminating tumors with IRs or CRs. (a) and (b) $\left[\mathrm{HbO}_{2}\right]$ at week 1 and week 3 in HPV-16 positive patients, (c) THC at week 3 in HPV16 positive patients, (d) $\mathrm{StO}_{2}$ at week 3 in HPV-16 positive patients, (e) BFI in HPV-16 negative patients at week 3 , and (f) $\mu_{\mathrm{s}}^{\prime}$ in HPV-16 negative patients at week 3 . 
Figure 5 shows corresponding ROC curves of these parameters for discriminating tumors with IRs or CRs.

Specifically in HPV-16 positive patients, $\left[\mathrm{HbO}_{2}\right]$ provided the best prediction of treatment outcomes at week 1 and week 3 [AUC $=0.87$ (95\% CI: 0.68 to 1.00)]. THC and $\mathrm{StO}_{2}$ also showed moderate predictions of treatment outcomes at week 3 [AUC $=0.83$ (95\% CI: 0.61 to 0.97 ) and $\mathrm{AUC}=0.84$ (95\% CI: 0.63 to 0.97 ), respectively]. In HPV-16 negative patients, BFI and $\mu_{\mathrm{s}}^{\prime}$ provided excellent predictions of treatment outcomes at week 3 [AUC $=0.93$ (95\% CI: 0.72 to 1.00$)$ and 0.96 (95\% CI: 0.84 to 1.00 ), respectively].

\subsection{Comparison of Tumor Hemodynamic Responses and Treatment Outcomes Between the HPV-16 Positive and Negative Patients}

Overall, there were no significant differences in most of the measured optical parameters between the HPV-16 positive and negative groups, except pretreatment baseline $[\mathrm{Hb}](p=0.001)$ in tumors. Post hoc pairwise tests demonstrated that the HPV-16 negative group had a significantly $(p=0.01)$ higher level in tumor $[\mathrm{Hb}](19.69 \pm 1.71 \mu \mathrm{M})$ at pretreatment baseline (week 0 ) compared to the HPV-16 positive group $(14.59 \pm 0.85 \mu \mathrm{M})$.

There were no significant differences between the HPV-16 positive and negative groups in age, gender, tumor stage, tumor node size, and cutaneous tissue thickness above the tumor, but significant difference was found in primary tumor site $(p<0.001)$.

An insignificantly $(p=1.00)$ higher frequency of $\mathrm{CR}$ (61.54\%, 95\% CI: $40.57 \%$ to $79.77 \%)$ was observed in HPV16 positive tumors than that in HPV-16 negative tumors (58.33\%, $95 \%$ CI: $27.67 \%$ to $84.83 \%)$.

\section{Discussion and Conclusions}

This study aimed to evaluate the accuracy of tumor hemodynamic parameters measured noninvasively by the optical techniques for early prediction of chemoradiation therapy outcomes in patients with head and neck cancer. For this purpose, we utilized a hybrid diffuse optical instrument developed in our laboratory and measured dynamic changes in optical properties and hemodynamics of local tumors and forearm muscles (as global controls for comparison) prior to the treatment and weekly over the treatment period of 7 weeks. We examined the predictive values of multiple parameters measured in the local tumors including BFI, $\mu_{\mathrm{s}}^{\prime},\left[\mathrm{HbO}_{2}\right],[\mathrm{Hb}], \mathrm{THC}$, and $\mathrm{StO}_{2}$ in relation to one-year treatment outcomes. In addition, we also evaluated the influence of other factors on treatment outcomes (i.e., IR or CR) including age, gender, tumor stage, tumor node size, cutaneous tissue thickness above the tumor, and HPV-16 status.

No significant baseline differences before treatment in age, gender, tumor site, tumor stage, tumor node size, cutaneous tissue thickness, HPV-16 status, and optical measurement parameters of local tumors and control muscles were observed between the IR and CR groups (see Table 1 and Fig. 2), suggesting the insignificant influence of these baseline factors on treatment outcomes. Forearm hemodynamics did not show significant difference between the IR and CR groups at any measurement time point before and during treatment, indicating insignificant impacts of chemoradiation therapy on tissues outside the radiation beam.

Based on the overall results from 47 patients, IR tumors exhibited significantly lower levels in $\left[\mathrm{HbO}_{2}\right], \mathrm{THC}$, and $\mathrm{StO}_{2}$ than CR tumors over the treatment period (see Fig. 2), which agrees with previous observations that HNSCCs with insufficient oxygen had poor treatment outcomes. ${ }^{9,10}$ However, post hoc pairwise tests could not find significant difference at any specific measurement time point (see Fig. 2), making it difficult to use these parameters measured at one time point to predict treatment outcomes. Interestingly, after breaking down the patients into two subgroups of HPV-16 positive and HPV-16 negative, post hoc pairwise tests found significant differences in some of the measured parameters at certain measurement time points between the IR and CR groups.

Specifically, in the subgroup of HPV-16 positive patients, IR tumors demonstrated significantly lower levels of $\left[\mathrm{HbO}_{2}\right]$ at weeks 1 to 3 as well as THC and $\mathrm{StO}_{2}$ at week 3, compared to CR tumors (see Fig. 3). By contrast, in the subgroup of HPV-16 negative patients, IR tumors showed significantly higher levels in BFI and $\mu_{\mathrm{s}}^{\prime}$ at week 3, compared to CR tumors (see Fig. 4). All these parameters measured during the early stage of the treatment (within the first three weeks of treatment) exhibited moderate to high accuracy in predicting one-year treatment outcomes within each subgroup (see Table 2 and Fig. 5). Note, however, that the pretreatment baseline levels of tumor hemodynamics measured at week 0 in each subgroup did not show any predictive values for treatment outcomes, indicating the importance of repeated monitoring of hemodynamic changes during therapy. In addition, because the number of HPV-16 positive tumors $(n=26)$ measured in this study is much larger than that of HPV-16 negative tumors $(n=12)$ (see Table 1$)$, it is not surprising that overall tumor hemodynamic responses (see Fig. 2) follow similar trends of hemodynamic changes in HPV-16 positive tumors (see Fig. 3).

Several clinical studies have shown that HPV positive HNSCCs have a more favorable outcome and greater response to radiotherapy than HPV negative HNSCCs. ${ }^{35-37}$ In this study, we observed an insignificantly higher frequency of having CRs in HPV-16 positive tumors compared to that in HPV-16 negative tumors (see Sec. 3.5). Such insignificance may be due to the relatively small number of patients enrolled in this study. However, it is unclear why and how HPV-16 status affects predictive capabilities of our measured parameters differently for predicting treatment outcomes. For example, we found in this study that $\left[\mathrm{HbO}_{2}\right], \mathrm{THC}$, and $\mathrm{StO}_{2}$ showed predictive values for treatment outcomes in HPV-16 positive tumors while BFI and $\mu_{\mathrm{s}}^{\prime}$ provided the best prediction of treatment outcomes in HPV-16 negative tumors. Although the differences between HPV-16 positive and negative tumors in gene mutations, ${ }^{38}$ hypoxia, ${ }^{39}$ angiogenesis, ${ }^{40}$ metabolic profile, ${ }^{41}$ and adaptation to hypoxia have been suggested to impact treatment outcomes, pathogenic mechanisms underlying these differences remain speculative or even contradictory.

Previous studies have demonstrated equal frequencies of baseline hypoxic tumors between the HPV-positive and HPVnegative patients, determined by Eppendorf electrode or PET/ $\mathrm{CT}^{42-44}$ The only significant differences observed in our study between the HPV-positive and HPV-negative patients were the baseline levels of $[\mathrm{Hb}]$ and primary tumor sites, which seemed to not significantly affect the treatment outcomes (see Sec. 3.5).

Only a few studies have monitored dynamic tumor oxygenation changes during the period of treatment in a small number of patients. ${ }^{11,43}$ For example, Mortensen et al. conducted 18F-fluorozzomycin arabinoside (FAZA) PET/CT hypoxia imaging in 
HNSCC patients treated with radiotherapy. ${ }^{43}$ Static imaging was obtained on all 40 patients prior to irradiation and the distribution of hypoxia among the HPV-positive and negative tumors was not significantly different. Only 13 of the 40 patients had a repeat FAZA PET/CT scan separated from the pretreatment imaging by 14 to 24 days. The repeat imaging showed a general decline in the hypoxic volume compared to the baseline hypoxia. In another pilot study using invasive polarographic needle electrodes, Lyng et al. ${ }^{11}$ measured tumor $\mathrm{pO}_{2}$ before treatment $(n=11)$ and once a week during therapy $(n=8)$. Significant fluctuations in $\mathrm{pO}_{2}$ occurred during radiotherapy, and results showed that progressive disease occurred among the patients with highly hypoxic tumors, regardless of hypoxic fractions before or after treatment. In addition to the limited numbers of patients studied, another limitation of both studies was that the authors did not report whether these hypoxic variations induced by the therapy were associated with HPV positivity.

In this study, we took advantage of a noninvasive and portable NIR diffuse optical technique to repeatedly monitor tumor hemodynamic changes over the long treatment period. Significant changes in tumor hemodynamics were found during the early stage of the treatment, which affected significantly the treatment outcomes. In the subgroup of HPV-16 positive tumors, $\left[\mathrm{HbO}_{2}\right], \mathrm{THC}$, and $\mathrm{StO}_{2}$ increased during the first three weeks of treatment, which were reasonably associated with complete tumor responses to therapy (see Fig. 3). In contrast, in the subgroup of HPV-16 negative tumors, there was no significant difference in local tumor oxygenation between the IR and CR groups during chemoradiation therapy (see Fig. 4). Instead, significantly higher levels of $\mu_{\mathrm{s}}^{\prime}$ and BFI were observed in HPV-16 negative tumors with IRs at week 3 . High angiogenesis (associated with high $\mu_{\mathrm{s}}^{\prime 45}$ ) was previously found to be related to poor local control rate in HNSCCs under radiotherapy. ${ }^{46,47}$ Dynamic increases in tumor blood flow during radiotherapy were also detected by dynamic CT and DCS in HNSCCs with IRs to therapy. ${ }^{13,15}$ Therefore, we speculate that the significantly higher levels of $\mu_{\mathrm{s}}^{\prime}$ and BFI observed in HPV-16 negative tumors with IRs may be associated with more active angiogenesis, which forms defective vascular networks, leading to blood leakiness inside tumors and reduced oxygen supply. In addition, a higher blood flow level may be attributed to a higher up-regulation of vascular endothelial growth factor (VEGF) in IR tumor cells than that in CR tumor cells, which promotes the survival of endothelial cells and thereby radiation resistance. ${ }^{48}$ Also, higher expression of endothelial nitric oxide in IR tumors may further enhance blood perfusion, open up nonperfused vessels, and promote neoangiogenesis. ${ }^{13,49}$ Further comprehensive investigations are needed to understand why and how HPV-16 status affects predictive values of different parameters for tumor responses to chemoradiation therapy.

Nevertheless, results from our study suggest that dynamic changes in tumor microenvironment during treatment significantly influence treatment outcomes, and frequent measurements of dynamic changes in tumor properties (e.g., $\mu_{\mathrm{s}}^{\prime}$ ) and hemodynamics (e.g., BFI, $\left[\mathrm{HbO}_{2}\right],[\mathrm{Hb}]$, THC, and $\mathrm{StO}_{2}$ ) during chemoradiotherapy generate essential information for early prediction of tumor responses to therapy. These hemodynamic parameters exhibited significantly high accuracy (AUCs ranging from 0.83 to 0.96 ) for early prediction of clinical outcomes within the first three weeks of therapy.

Such predications, based on frequent optical measurements, may ultimately be used to optimize individual therapeutic outcomes at an early time of radiotherapy. For example, treatment outcomes may be improved by dynamically promoting oxygenation levels (e.g., hyperbaric oxygen therapy) in HPV16 positive tumors or inhibiting angiogenesis (e.g., antiVEGF antibody) in HPV-16 negative tumors. ${ }^{50}$

In summary, our diffuse optical measurements show the promise for early (within the first 3 weeks of treatment) prediction of chemoradiation therapy in patients with head and neck cancer. Since HPV-16 status affects tumor response to therapy, subgrouping patients based on HPV-16 status is necessary to improve the predictive accuracy for treatment outcomes. More patients are needed to increase statistical power of data analysis, to develop a predictive model integrating multiple parameters for further improving predictive accuracy, and to find optimal thresholds of the parameters for validating the classifier. In addition, patients who are predicted to have an IR to chemoradiation may be guided to alternative therapies (e.g., surgical removal of tumors) resulting in a reduction of the side effects associated with chemoradiation. Dynamic modification of tumor properties/hemodynamics based on frequent optical measurements during chemoradiation therapy is also expected to optimize treatment outcomes individually.

\section{Acknowledgments}

This work was partially supported by the National Institutes of Health under Grant R01 CA149274 (GY), Grant R21 AR062356 (GY), Grant UL1RR033173 (GY), and the Biostatistics and Bioinformatics Shared Resource Facility of the University of Kentucky Markey Cancer Center (P30CA177558). We thank Dr. Tadahide Izumi for constructive discussion. We also thank Daniel Kameny, Jacqueline Sims, Karen Meekins, Laura Reichel, and Marta Wood for their assistance in recruitment of patients.

\section{References}

1. R. I. Haddad, Multidisciplinary Management of Head and Neck Cancer, Demos Medical, New York (2011).

2. A. Argiris et al., "Head and neck cancer," Lancet 371(9625), 1695-1709 (2008).

3. A. R. Kreimer et al., "Human papillomavirus types in head and neck squamous cell carcinomas worldwide: a systematic review," Cancer Epidemiol. Biomarkers Prev. 14(2), 467-475 (2005).

4. M. H. Barcellos-Hoff, C. Park, and E. G. Wright, "Radiation and the microenvironment-tumorigenesis and therapy," Nat. Rev. Cancer 5(11), 867-875 (2005).

5. R. E. Lenhard et al., Clinical Oncology, 1st ed., American Cancer Society, Atlanta, Georgia (2001).

6. V. T. DeVita, T. S. Lawrence, and S. A. Rosenberg, DeVita, Hellman, and Rosenberg's Cancer: Principles \& Practice of Oncology, 9th ed., Wolters Kluwer Health/Lippincott Williams \& Wilkins, Philadelphia (2011).

7. E. J. Hall and A. J. Giaccia, Radiobiology for the Radiologist, 7th ed., Wolters Kluwer Health/Lippincott Williams \& Wilkins, Philadelphia (2012).

8. R. A. Gatenby et al., "Oxygen distribution in squamous cell carcinoma metastases and its relationship to outcome of radiation therapy," Int. J. Radiat. Oncol., Biol., Phys. 14(5), 831-838 (1988).

9. M. Nordsmark, M. Overgaard, and J. Overgaard, "Pretreatment oxygenation predicts radiation response in advanced squamous cell carcinoma of the head and neck," Radiother. Oncol.: J. Eur. Soc. Ther. Radiol. Oncol. 41(1), 31-39 (1996).

10. D. M. Brizel et al., "Tumor hypoxia adversely affects the prognosis of carcinoma of the head and neck," Int. J. Radiat. Oncol., Biol., Phys. 38(2), 285-289 (1997). 
11. H. Lyng et al., "Changes in oxygen tension during radiotherapy of head and neck tumours," Acta Oncologica 38(8), 1037-1042 (1999).

12. K. Lehtio et al., "Imaging perfusion and hypoxia with PET to predict radiotherapy response in head-and-neck cancer," Int. J. Radiat. Oncol., Biol., Phys. 59(4), 971-982 (2004).

13. K. Surlan-Popovic et al., "Changes in perfusion CT of advanced squamous cell carcinoma of the head and neck treated during the course of concomitant chemoradiotherapy," Am. J. Neuroradiol. 31(3), 570-575 (2010).

14. S. B. Donaldson et al., "Perfusion estimated with rapid dynamic contrast-enhanced magnetic resonance imaging correlates inversely with vascular endothelial growth factor expression and pimonidazole staining in head-and-neck cancer: a pilot study," Int. J. Radiat. Oncol., Biol., Phys. 81(4), 1176-1183 (2011).

15. U. Sunar et al., "Noninvasive diffuse optical measurement of blood flow and blood oxygenation for monitoring radiation therapy in patients with head and neck tumors: a pilot study," J. Biomed. Opt. 11(6), 064021 (2006).

16. L. Dong et al., "Noninvasive diffuse optical monitoring of head and neck tumor blood flow and oxygenation during radiation delivery," Biomed. Opt. Express 3(2), 259-272 (2012).

17. D. Irwin et al., "Influences of tissue absorption and scattering on diffuse correlation spectroscopy blood flow measurements," Biomed. Opt. Express 2(7), 1969-1985 (2011).

18. L. Dong et al., "Simultaneously extracting multiple parameters via fitting one single autocorrelation function curve in diffuse correlation spectroscopy," IEEE Trans. Biomed. Eng. 60(2), 361-368 (2013).

19. Y. Shang et al., "Portable optical tissue flow oximeter based on diffuse correlation spectroscopy," Opt. Lett. 34(22), 3556-3558 (2009).

20. K. Gurley, Y. Shang, and G. Yu, "Noninvasive optical quantification of absolute blood flow, blood oxygenation, and oxygen consumption rate in exercising skeletal muscle," J Biomed Opt 17(7), 075010 (2012).

21. A. Becker et al., "Oxygenation of squamous cell carcinoma of the head and neck: comparison of primary tumors, neck node metastases, and normal tissue," Int. J. Radiat. Oncol., Biol., Phys. 42(1), 35-41 (1998).

22. S. Fantini, M. A. Franceschini, and E. Gratton, "Semi-infinite-geometry boundary-problem for light migration in highly scattering media - a frequency-domain study in the diffusion-approximation," J. Opt. Soc. Am. B 11(10), 2128-2138 (1994).

23. S. A. Carp et al., "Hemodynamic signature of breast cancer under fractional mammographic compression using a dynamic diffuse optical tomography system," Biomed. Opt. Express 4(12), 2911-2924 (2013).

24. D. A. Boas and A. G. Yodh, "Spatially varying dynamical properties of turbid media probed with diffusing temporal light correlation," J. Opt. Soc. Am. A 14(1), 192-215 (1997).

25. C. Cheung et al., "In vivo cerebrovascular measurement combining diffuse near-infrared absorption and correlation spectroscopies," Phys. Med. Biol. 46(8), 2053-2065 (2001).

26. J. Li et al., "Transient functional blood flow change in the human brain measured noninvasively by diffusing-wave spectroscopy," Opt. Lett. 33(19), 2233-2235 (2008).

27. S. O. Rice, "Mathematical analysis of random noise," in Noise and Stochastic Processes N. Wax, Ed., p. 133, Dover, New York (1954).

28. G. Yu et al., "Validation of diffuse correlation spectroscopy for muscle blood flow with concurrent arterial spin labeled perfusion MRI," Opt. Express 15(3), 1064-1075 (2007).

29. G. Yu et al., "Noninvasive monitoring of murine tumor blood flow during and after photodynamic therapy provides early assessment of therapeutic efficacy," Clin. Cancer Res. 11(9), 3543-3552 (2005).

30. T. Durduran et al., "Diffuse optical measurement of blood flow in breast tumors," Opt. Lett. 30(21), 2915-2917 (2005).

31. C. Zhou et al., "Diffuse optical monitoring of blood flow and oxygenation in human breast cancer during early stages of neoadjuvant chemotherapy," J. Biomed. Opt. 12(5), 051903 (2007).

32. K. T. Kuo et al., "The biomarkers of human papillomavirus infection in tonsillar squamous cell carcinoma-molecular basis and predicting favorable outcome," Mod. Pathol. 21(4), 376-386 (2008).

33. P. Diggle and P. Diggle, Analysis of Longitudinal Data, 2nd ed., Oxford University Press, Oxford, New York (2002).

34. B. Efron and R. Tibshirani, An Introduction to the Bootstrap, Chapman \& Hall, New York (1993).
35. P. Lassen et al., "Effect of HPV-associated p16INK4A expression on response to radiotherapy and survival in squamous cell carcinoma of the head and neck," J. Clin. Oncol. 27(12), 1992-1998 (2009).

36. C. Fakhry et al., "Improved survival of patients with human papillomavirus-positive head and neck squamous cell carcinoma in a prospective clinical trial," J. Natl. Cancer Inst. 100(4), 261-269 (2008).

37. F. Petrelli, E. Sarti, and S. Barni, "Predictive value of human papillomavirus in oropharyngeal carcinoma treated with radiotherapy: an updated systematic review and meta-analysis of 30 trials," Head Neck 36(5), 750-759 (2014).

38. T. Y. Seiwert et al., "Integrative and comparative genomic analysis of HPV-positive and HPV-negative head and neck squamous cell carcinomas," Clin. Cancer Res. 21(3), 632-641 (2015).

39. L. Sepiashvili et al., "Novel insights into head and neck cancer using next-generation 'omic' technologies," Cancer Res. 75(3), 480-486 (2015).

40. J. D. Troy et al., "Expression of EGFR, VEGF, and NOTCH1 suggest differences in tumor angiogenesis in HPV-positive and HPV-negative head and neck squamous cell carcinoma," Head Neck Pathol. 7(4), 344-355 (2013).

41. R. Krupar et al., "Immunologic and metabolic characteristics of HPVnegative and HPV-positive head and neck squamous cell carcinomas are strikingly different," Virchows Arch. 465(3), 299-312 (2014).

42. B. S. Sorensen et al., "Radiosensitivity and effect of hypoxia in HPV positive head and neck cancer cells," Radiother. Oncol. 108(3), 500-505 (2013).

43. L. S. Mortensen et al., "FAZA PET/CT hypoxia imaging in patients with squamous cell carcinoma of the head and neck treated with radiotherapy: results from the DAHANCA 24 trial," Radiother. Oncol. 105(1), 14-20 (2012).

44. C. S. Kong et al., "The relationship between human papillomavirus status and other molecular prognostic markers in head and neck squamous cell carcinomas," Int. J. Radiat. Oncol., Biol., Phys. 74(2), 553-561 (2009).

45. V. R. Kondepati, H. M. Heise, and J. Backhaus, "Recent applications of near-infrared spectroscopy in cancer diagnosis and therapy," Anal. Bioanal. Chem. 390(1), 125-139 (2008).

46. M. I. Koukourakis et al., "Angiogenesis, thymidine phosphorylase, and resistance of squamous cell head and neck cancer to cytotoxic and radiation therapy," Clin. Cancer Res. 6(2), 381-389 (2000).

47. M. I. Koukourakis et al., "Hypoxia-regulated carbonic anhydrase-9 (CA9) relates to poor vascularization and resistance of squamous cell head and neck cancer to chemoradiotherapy," Clin. Cancer Res. 7(11), 3399-3403 (2001).

48. V. K. Gupta et al., "Vascular endothelial growth factor enhances endothelial cell survival and tumor radioresistance," Cancer J. 8(1), 47-54 (2002).

49. D. Koutsimpelas et al., "Proangiogenic effects of ionizing irradiation on squamous cell carcinoma of the hypopharynx," Auris Nasus Larynx 35(3), 369-375 (2008).

50. M. Yoshimura et al., "Microenvironment and radiation therapy," BioMed Res. Int. 2013, 685308 (2013).

Lixin Dong is an assistant professor of bioinformatics in the College of Nursing at Augusta University. He received his PhD in biomedical engineering at the University of Kentucky in 2015. His $\mathrm{PhD}$ research focused on developing novel diffuse optical technologies for monitoring tumor hemodynamic changes during chemoradiation treatment. His current research interest includes epigenetics, gene-environment interaction, and dietary intervention.

Mahesh Kudrimoti is a professor in the Department of Radiation Medicine at the University of Kentucky. His research interest is the development and application of novel treatment strategies in complex head/neck cancers and conduct of clinical/translational studies in cancer research. Currently, he is a member of the Head/Neck Cancer Research Team at the Markey Cancer Center and has several funded studies ongoing. $\mathrm{He}$ is the clinical lead on this paper.

Daniel Irwin is a graduate student in the Department of Biomedical Engineering at the University of Kentucky. He has been working on the development of noncontact diffuse correlation spectroscopy/ tomography and speckle contrast diffuse correlation spectroscopy 
Dong et al.: Diffuse optical measurements of head and neck tumor hemodynamics for early prediction...

for noninvasive assessment of blood flow contracts in animal and human tumors.

Li Chen is an associate professor in the Division of Cancer Biostatistics, Department of Biostatistics and the Biostatistics and Bioinformatics Shared Resource Facility at the Markey Cancer Center, University of Kentucky. She received her PhD in biostatistics from the University of North Carolina at Chapel Hill in 2009. Her research interest is the development and application of novel statistical methods for medical and epidemiological studies especially in cancer research. Specifically, she is interested in semiparametric and nonparametric methods, survival analysis, longitudinal data analysis, and prediction models.

Sameera Kumar is a current PGY-4 in the Department of Radiation Oncology at the University of Kentucky. Her current research interests include the change in hemodynamic parameters in tissues and tumors in response to treatment and exploring the use and efficacy of stereotactic body radiation therapy. Her role in this study was clinical data compilation and analysis of outcomes.

Yu Shang received his $\mathrm{PhD}$ in biomedical engineering in 2008. He was a research scientist working in the Department of Biomedical Engineering at the University of Kentucky and is now working as a faculty member in the Department of Biomedical Engineering at the North University of China. His current research interests focus on the development of near-infrared diffuse optical spectroscopy and diffuse correlation spectroscopy for noninvasive assessment of microvasculature blood flow/oxygenation/oxygen metabolism in biological tissues.

Chong Huang received his $\mathrm{PhD}$ in optical engineering in 2011 . He is a postdoctoral scholar in the Department of Biomedical Engineering at the University of Kentucky. His current research focuses on the development of noncontact diffuse correlation spectroscopy/tomography and speckle contrast diffuse correlation spectroscopy for noninvasive assessment of blood flow and oxygenation in biological tissues. His specialties include optical/mechanical system design, signal processing, finite element analysis, physiological modeling, data collection, and image reconstruction.

Ellis L. Johnson is a clinical medical physics professor in the Department of Radiation Medicine at the University of Kentucky. He also serves as the director of graduate studies for the Radiation Sciences Program and mentor in the medical physics residency. His research interests include radiation dosimetry and treatment planning methodologies, treatment methods in radiation therapy, and imaging for radiation therapy.

Scott D. Stevens is an associate professor in the Department of Radiology at the University of Kentucky. He has over 25 years of experience in ultrasound imaging.

Brent J. Shelton is professor of cancer biostatistics in the $\mathrm{NCl}$ Designated Markey Cancer Center at the University of Kentucky. He has nearly 25 years of experience in leading biostatistical efforts for research intensive projects. His predominant areas of collaborative research are focused on cancer prevention and control and in particular in the design, conduct, and analysis of cancer behavioral interventions, cancer biology basic science research, and cancer experimental therapeutics.

Guoqiang $\mathrm{Yu}$ is a professor in the Department of Biomedical Engineering at the University of Kentucky. He has over 20-years experience in the field of biomedical engineering and is currently leading a research group to develop various near-infrared diffuse spectroscopy and tomography systems for noninvasive imaging of deep tissue hemodynamics in animals and humans. 